\title{
Estimation of the outcrossing rate in the chestnut blight fungus, Cryphonectria parasitica
}

\author{
MICHAEL G. MILGROOM ${ }^{1 *}$, SUSANNE E. LIPARI ${ }^{1}$, RICHARD A. ENNOS $^{2} \&$ \\ YIR-CHUNG LIU' \\ ${ }^{1}$ Department of Plant Pathology, Cornell University, 334 Plant Science Building, Ithaca, NY 14853-5908, U.S.A. and \\ ${ }^{2}$ Institute of Ecology and Resource Management, University of Edinburgh, The King's Buildings, Mayfield Road, \\ Edinburgh EH9 3JU, U.K.
}

\begin{abstract}
The outcrossing rate in the chestnut blight fungus, Cryphonectria (Endothia) parasitica, was estimated in a natural population using data from six unlinked restriction fragment length polymorphism (RFLP) loci and DNA fingerprinting. This fungus was shown to have a mixed mating system with both self-fertilization and outcrossing in the same population. In a sample of 22 perithecia, all progeny segregated in 1:0,1:1 or $0: 1$ ratios at RFLP loci; there was no evidence for multiple male parents in ascospore families from single perithecia. The multilocus estimate of the outcrossing rate was 0.74 for the RFLP data; the proportion of perithecia with progeny segregating for fingerprint fragments was 0.73 . Vegetative compatibility was not a sensitive marker for detecting outcrossing as only 11 perithecia ( 50 per cent) showed segregation with this marker, compared with 16 ( 73 per cent) for DNA fingerprinting. We are not aware of any other report of a mixed mating system of a fungus in a natural population with an outcrossing rate significantly different from 1.0 or 0.0 .
\end{abstract}

Keywords: Cryphonectria parasitica, Endothia parasitica, heterothallism, homothallism, mating system, outcrossing.

\section{Introduction}

Mating systems can have a profound effect on genetic variability and population structure (Allard, 1975; Clegg, 1980; Hedrick, 1990). Recently, there has been a great deal of interest in mating systems in plant populations. One reason for this is that many plant populations deviate from assumptions of random mating which are commonly made in theoretical models of genetic transmission (Clegg, 1980). Plants exhibit a variety of mating systems which include selffertilization, outcrossing, or a mixture of both. The consequences of these different mating systems will markedly affect the structure and evolution of populations (Allard, 1975; Clegg, 1980; Hedrick, 1990).

In contrast to plants, however, there have been relatively few studies of fungal mating systems in natural populations. Fungi are usually classified as either heterothallic or homothallic. In heterothallic fungi sexual development can only occur after fertilization

*Correspondence. between compatible strains; homothallic species can reproduce sexually by self-fertilization (Fincham et al., 1979). Some homothallic species can also outcross in the laboratory (Fincham et al., 1979); this is known as 'relative heterothallism' (Hemmons et al., 1958; Beraha \& Garber, 1985). Other fungi appear to be primarily heterothallic but will rarely self-fertilize to produce viable offspring (Puhalla \& Anagnostakis, 1971; Brasier \& Gibbs, 1975). Thus, in some fungi there is a potential for mixed mating systems in nature, just as in flowering plants where both selfing and outcrossing can occur in the same population (e.g. Brown et al., 1975).

Most studies on mating systems in fungi have examined mating processes in culture. Laboratory studies may determine whether a species can be selffertilized or outcrossed, but do not provide any information on the frequency of these events in natural populations (Ennos \& Swales, 1987). Furthermore, laboratory studies cannot be performed on species which do not reproduce sexually in culture; the only approach to studying mating systems in these species is to study natural populations (Ennos \& Swales, 1987). 
Natural populations of fungi where mating systems have been investigated have been described as either predominantly outcrossing (Ainsworth, 1987; Ennos \& Swales, 1987; Sharland \& Rayner, 1989; Adams et al., 1990) or predominantly selfing (Rayner \& Turton, 1982; Ainsworth, 1987; Sharland et al., 1988; Sharland \& Rayner, 1989; Kohn et al., 1991; Kohli \& Kohn, 1992). However, a mixed mating system may be operating in a population of the chestnut blight fungus, Cryphonectria parasitica (formerly Endothia parasitica). Genetic analyses of $C$. parasitica auxotrophs showed that self-fertilization sometimes occurs (Puhalla \& Anagnostakis, 1971). When chestnut stems in a natural population were inoculated with genetically marked strains of $C$. parasitica, more than half the perithecia sampled from seven stems were self-fertilized after 21 months (S. L. Anagnostakis, personal communication). The remaining perithecia were thought to have outcrossed with naturally occurring strains in the population.

An important question to address for understanding the mating system of $C$. parasitica is the extent to which outcrossing occurs in a natural population. The primary objective of this research was to estimate quantitatively the outcrossing rate of $C$. parasitica in a natural population. We also had several subsidiary objectives. The first was to investigate the inheritance and linkage of the restriction fragment length polymorphisms (RFLPs) we used for genetic markers. Second, to understand the mating system of $C$. parasitica in natural populations we looked for evidence of multiple fertilizations giving rise to progeny in each fruit body (perithecium). Finally, we compared three different types of data for estimating outcrossing: RFLPs, DNA fingerprinting and vegetative compatibility groups.

\section{Materials and methods}

\section{Study organism}

The biology and genetics of $C$. parasitica were recently reviewed by Anagnostakis $(1987,1988)$. C. parasitica is an ascomycete fungus that causes chestnut blight on Castanea spp. The fungus produces haploid vegetative mycelium that grows through the bark, destroying the cambial tissues and causing cankers. Two types of spores are produced in the cankers: asexual spores (conidia) and sexual spores (ascospores). Conidia are exuded in sticky tendrils and are dispersed by water, insects, birds or other animals. Conidia can function either as vegetative propagules that cause new cankers when dispersed to wounds in the bark, or as male gametes that fertilize receptive hyphae in existing cankers. When fertilization occurs, dikaryotic ascogenous hyphae arise which later form numerous asci in fruit bodies called perithecia. Meiosis occurs in asci resulting in haploid ascospores which are forcibly discharged from the perithecium and dispersed by wind to initiate new cankers. Like many ascomycetes, C. parasitica has two mating types. In the laboratory, crossing only occurs between strains of different mating types; however, self-fertilization does occur rarely.

\section{Population sample}

The population of $C$. parasitica we sampled for this study has previously been analysed for population structure using DNA fingerprinting (Milgroom et al., 1992a). The study site is located in the Appalachian mountains at the Mountain Lake Biological Station, Giles County, VA, U.S.A. A $25 \times 25 \mathrm{~m}$ plot was marked out in a mixed-oak hardwood forest that had formerly been covered primarily by American chestnut until blight destroyed all of them in the 1930s. Bark samples $\left(2-5 \mathrm{~cm}^{2}\right)$ were collected from the margin of every blight canker visible on chestnut sprouts within this plot. We recovered a total of 39 C. parasitica mass hyphal isolates, one from each canker, in this sample using methods described by Anagnostakis (1988). In addition to canker isolates, we collected at least 10 random ascospores from each of 22 perithecia from 17 different cankers. Ascospores were collected by peeling the top layers of bark and stromatal tissue from the canker samples to reveal the undersides of perithecia. A single perithecium was then pierced with a sterile needle and a droplet containing ascospores was transferred to a droplet of sterile water on 4 per cent water agar in a Petri dish. Ascospores were spread on the surface of the agar with a sterile loop and incubated at $25^{\circ} \mathrm{C}$ for $18-24 \mathrm{~h}$. Single germinated ascospores were then transferred to potato dextrose agar (Difco, PDA) with a fine needle. Mycelium for DNA extraction was cultured as described previously (Milgroom et al., 1992a,b).

\section{Electrophoresis and Southern blot analysis}

We used two types of probes for Southern blot analysis in this study. A DNA fingerprinting probe, pMS5.1 (Milgroom et al., 1992a), was used which hybridizes to 7-12 restriction fragments in each isolate of $C$. parasitica giving unique patterns for 33 of the 39 canker isolates in this population. We also used four probes that hybridized to fewer fragments and are putatively single- or low-copy in the $C$. parasitica genome; for convenience we refer to these probes as RFLP probes to distinguish them from the fingerprinting 
ing probe. Three RFLP probes were found by screening randomly selected phage lambda clones from a library constructed by Powell \& Van Alfen (1987). Two of these were subcloned into plasmids pMS11.1 and pMS29.1 using pUC19 as a vector; the third was subcloned into pMS26.3 in a Bluescript IIKS (Stratagene) vector. We also used a probe ( $\mathrm{pCB} 15$ ) previously identified from a plasmid library that showed polymorphism in $C$. parasitica isolates from the U.S.A. (Milgroom et al., 1992b). We used the same techniques for DNA preparation, electrophoresis and Southern blot analysis as were described by Milgroom et al. (1992a,b). All restriction digests were performed with the restriction endonuclease PstI.

\section{Inheritance of RFLP markers}

We analysed the inheritance and linkage of the RFLPs using the same progeny used for previously analysing DNA fingerprints (Milgroom et al., 1992a). Random ascospore progeny were collected from a single perithecium of a controlled laboratory cross (43 progeny) and from one of the perithecia (V8-1) collected from a canker in the Mt. Lake field population (49 progeny). For each progeny set we tested whether the polymorphic fragments hybridizing to each probe segregated in 1:1 ratios. We tested for linkage among segregating fragments from the different probes using chi-square tests for independence. RFLP data for 8-10 progeny from each of the remaining 21 perithecia from the Mt. Lake population were also used for analysing inheritance.

\section{Vegetative compatibility testing}

In addition to molecular markers we also used vegetative compatibility (VC) as a phenotypic marker to determine if progeny from each perithecium were the result of outcrossing. Vegetative compatibility is controlled by five to seven unlinked vegetative incompatibility (vic) loci (Anagnostakis, 1982a, 1988); individuals are compatible only if they share the same alleles at all vic loci. We tested 10-20 progeny from each perithecium to determine how many were in the same VC group as the maternal canker isolate. VC testing was done as described by Anagnostakis (1988): small cubes of agar $(\sim 3 \mathrm{~mm}$ on each side) were cut from the margins of actively growing progeny colonies and placed on PDA next to a similar piece of agar with mycelium from the maternal isolate. Test plates were incubated at $25^{\circ} \mathrm{C}$ in the dark for $6-7$ days before being scored. The presence of a barrage line was taken as evidence for incompatibility (Anagnostakis, 1988). Each test was performed twice.

\section{DNA fingerprinting}

Probe pMS5.1 was used to determine DNA fingerprints (Milgroom et al., 1992a) of canker isolates and progeny to determine whether segregation was occurring within each ascospore family. For fingerprint analysis of progeny we used a two-stage procedure. First we screened four or five randomly selected progeny from each family and determined whether segregation for any fingerprinting fragment could be detected. If no segregation was detectable, we analysed another four or five progeny from the same family for a total of at least eight. Assuming 1:1 segregation, as demonstrated previously (Milgroom et al., 1992a), with eight progeny there would be a probability greater than 0.99 of detecting outcrossing for each segregating locus. An important assumption in this approach was that mutation was negligible and would not be confused with segregation of small progeny numbers. DNA fingerprints have been shown previously to be relatively stable (Milgroom et al., 1992a).

\section{Results}

\section{Inheritance of RLFP markers}

All four RFLP probes we used revealed polymorphisms in this population (Table 1). Probes pMS11.1 and pMS29.1 hybridized to single loci, MS11 and MS29, respectively; each locus had two alleles with restriction fragment sizes of 5.3 or 3.5 kilobases $(\mathrm{kb})$ for MS11, and 8.0 or $6.2 \mathrm{~kb}$ for $M S 29$. There were four different polymorphic fragments at locus MS26 (hybridizing to pMS 26.3); these were approximately $1.7,1.5,1.4$ or $1.3 \mathrm{~kb}$ in length. Probe pCB15 hybridized to three polymorphic loci; these were designated: $C B 15-1$ with

Table 1 Estimates of allele frequencies in the Mt. Lake population of $C$. parasitica for six $\operatorname{RFLP} \operatorname{loci}(n=39)$

\begin{tabular}{|c|c|c|c|c|}
\hline \multirow[b]{2}{*}{ Locus } & \multicolumn{4}{|c|}{ Alleles $^{\mathrm{a}}$} \\
\hline & 1 & 2 & 3 & 4 \\
\hline$M S 11$ & 0.128 & 0.872 & - & - \\
\hline MS26 & 0.641 & 0.256 & 0.077 & 0.026 \\
\hline$M S 29$ & 0.692 & 0.308 & - & - \\
\hline$C B 15-1$ & 0.821 & 0.179 & - & - \\
\hline$C B 15-2$ & 0.769 & 0.231 & - & - \\
\hline$C B 15-3$ & 0.949 & 0.051 & - & - \\
\hline
\end{tabular}

${ }^{a}$ Alleles are ordered from largest to smallest polymorphic fragments (see text for sizes in kilobases). Alleles for $C B 15-3$ are ordered for presence then absence of a $3.3 \mathrm{~kb}$ fragment. 
alternate restriction fragments of 2.5 or $2.0 \mathrm{~kb}, C B 15-2$ with fragments of 2.2 or $1.8 \mathrm{~kb}$, and $C B 15-3$ which had the presence or absence of a $3.3 \mathrm{~kb}$ fragment. No attempts were made to determine whethe: polymorphisms were due to restriction site or length differences.

All six RFLP loci showed simple Mendelian inheritance. Five of the polymorphic loci segregated in $1: 1$ ratios in the laboratory cross $(n=43)$ or in the 49 ascospore isolates from a single field-collected perithecium (Table 2). The only inheritance data available for MS11 were from small progeny arrays where ratios were $5: 5$ and 4:6 for two perithecia where segregation occurred. No significant $(P>0.10)$ linkage was detected between any loci segregating in the same progeny set (Table 3 ); with progeny sizes of 43 and 49 , we would be able to detect linkage of approximately 35 map units or less at the 0.05 level of significance.

There are two lines of evidence that suggest that each perithecium produced progeny after fertilization by a single conidial parent. First, all but one of the segregation ratios were not different from 1:0, 1:1 or $0: 1(P>0.10)$ (Table 4). One family (perithecium V82 ), in which segregation occurred for two loci, had a ratio of 1:7 (Table 4) for locus MS26; this is expected to occur by chance with a probability of 0.035 if there is $1: 1$ segregation and is thus not unlikely to occur by chance when examining a large number of ratios. The second type of evidence in support of single fertilization is that only two alleles segregate at locus MS26 even though there are four alleles present in the popu-

Table 2 Segregation ratios at RFLP loci in C. parasitica

\begin{tabular}{lcc}
\hline \multicolumn{1}{l}{ Locus } & \multicolumn{1}{c}{ Ratio } & \multicolumn{1}{c}{$X_{(1)^{2}}^{\mathrm{al}}$} \\
\hline Laboratory cross perithecium $(X 2-1)$ & \\
MS26 & $19: 23^{\mathrm{b}}$ & 0.381 \\
MS29 & $23: 20$ & 0.209 \\
CB15-1 & $23: 20$ & 0.209 \\
CB15-3 & $24: 19$ & 0.581 \\
Field-collected perithecium & $($ V8-1) & \\
MS26 & $24: 25^{\mathrm{c}}$ & 0.020 \\
CB15-2 & $26: 23$ & 0.184 \\
\hline
\end{tabular}

${ }^{\mathrm{a}} \chi^{2}$ statistic ( 1 d.f.) for testing the null hypothesis of $1: 1$ segregation.

bAlleles are ordered from larger to smaller polymorphic fragment; or presence then absence of a fragment for locus CB15-3. 1.5 and $1.4 \mathrm{~kb}$ alleles segregated at locus MS26 in perithecium $X 2-1$.

" 1.4 and $1.3 \mathrm{~kb}$ alleles segregated at locus MS26 in perithecium V8-1. lation. If multiple paternity, which has been observed in laboratory crosses (Anagnostakis, 1982b), were common in this population then single perithecia would segregate occasionally for three or possibly four alleles, and some segregation ratios would be expected to deviate from $1: 0,1: 1$ or $0: 1$.

\section{Estimation of the outcrossing rate}

Having ascertained that all progeny within a perithecium are products of a single fertilization, we used this fact for estimating the extent to which self-fertilization occurs. The most appropriate parameter for measuring the mating system is, therefore, the proportion of perithecia that are formed following outcrossing, which we call $t$.

From data available at all the RFLP loci, we used a multilocus estimate of the outcrossing rate, $\hat{t}_{\mathrm{m}}$, as described by Shaw et al. (1981) but modified for haploid populations. In this method, the observed number of discernible outcrosses, i.e. the number of perithecia for which progeny segregate for at least one RFLP locus (Table 4), is compared with the number of expected discernible outcrosses under random mating $(t=1.0)$ given the actual frequency of alleles and maternal genotypes in the population scored. The multilocus probability that we would fail to detect an outcross event for a haploid population is given by

$\hat{\alpha}=\prod_{j}\left[\sum_{i} p_{\mathrm{ij}} p_{\mathrm{m}, \mathrm{ij}}\right]$

Table 3 Tests for independence in segregation between different RFLP loci in C. parasitica

\begin{tabular}{llrlllll}
\hline Loci & $A_{1} B_{1}{ }^{a}$ & $A_{1} B_{2}$ & $A_{2} B_{l}$ & $A_{2} B_{2}$ & $\chi_{(1)}^{2}$ \\
\hline \multicolumn{7}{l}{ Laboratory cross perithecium $(X 2-1)$} \\
MS26 & MS29 & 13 & 6 & 10 & 13 & 2.613 \\
MS26 & $C B 15-1$ & 9 & 9 & 13 & 10 & 0.004 \\
MS26 & $C B 15-3$ & 10 & 8 & 13 & 10 & 0.173 \\
MS29 & $C B 15-1$ & 12 & 11 & 11 & 8 & 0.137 \\
MS29 & $C B 15-3$ & 12 & 11 & 11 & 8 & 0.137 \\
CB15-1 & $C B 15-3$ & 11 & 12 & 12 & 7 & 0.987
\end{tabular}

Field-collected perithecium (V8-1)

$\begin{array}{lllllll}M S 26 & C B-2 & 14 & 10 & 12 & 13 & 0.525\end{array}$

"Numbers of progeny in four genotypic classes for each pair of loci. The $A$ and $B$ loci refer to the loci in the left and right columns, respectively, on each line. Alleles 1 and 2 are ordered from larger to smaller polymorphic fragment; or presence then absence of a fragment for locus CB15-3. 
Table 4 Segregation ratios for six RFLP loci for random ascospore families from 22 field-collected perithecia of $C$. parasitica from Mt. Lake Biological Station, VA, U.S.A. Segregation data for DNA fingerprinting and vegetative compatibility are also shown

\begin{tabular}{|c|c|c|c|c|c|c|c|c|c|}
\hline \multirow[b]{2}{*}{ Perithecium } & \multicolumn{6}{|c|}{ RFLP loci } & \multicolumn{3}{|c|}{ Segregation $^{\mathrm{a}}$} \\
\hline & $M S 11$ & $M S 26$ & $M S 29$ & $C B 15-1$ & $C B 15-2$ & CB15-3 & RFLP & VC & $\mathrm{fp}$ \\
\hline V5-5 & $0: 9^{\mathrm{b}}$ & $0: 9: 0: 0$ & $\underline{4}: 5^{\mathrm{c}}$ & 9.0 & $0: 9$ & $9: 0$ & + & + & + \\
\hline V7-4 & $0: 8$ & $3: 5: 0: 0$ & $\overline{4}: \underline{4}$ & $8: 0$ & $5: \underline{3}$ & $8: 0$ & + & + & + \\
\hline V7-6 & $0: 9$ & $0: 9: 0: 0$ & $4: \underline{5}$ & $9: 0$ & $0: \overline{9}$ & $9: 0$ & + & + & + \\
\hline V8-1 & $0: 49$ & $0: 0: 24: 25$ & 49:0 & $49: 0$ & $\underline{26}: 23$ & $49: 0$ & + & + & + \\
\hline V8-2 & $0: 8$ & $0: 0: 1: 7^{d}$ & $8: 0$ & $8: 0$ & $4: 4$ & $8: 0$ & + & + & + \\
\hline V11-1 & $0: 8$ & $8: 0: 0: 0$ & $6 . \underline{2}$ & $8: 0$ & $\underline{3}: 5$ & $8: 0$ & + & $-\mathrm{e}$ & + \\
\hline V13-1 & $0: 10$ & $10: 0: 0: 0$ & $7: \underline{2}$ & $4: \underline{6}$ & $10: 0$ & $10: 0$ & + & - & + \\
\hline V15-1 & $0: 9$ & $0: 0: 9: 0$ & $0: \overline{9}$ & $9: 0$ & $9: 0$ & $9: 0$ & - & - & - \\
\hline V17-1 & $5: 5$ & $10: 0: 0: 0$ & $10: 0$ & $10: 0$ & $10: 0$ & $10: 0$ & + & + & + \\
\hline V19-1 & $0: 10$ & $10: 0: 0: 0$ & $10: 0$ & $10: 0$ & $10: 0$ & $7: 3$ & + & + & + \\
\hline V19-2 & $0: 10$ & $10: 0: 0: 0$ & $10: 0$ & $10: 0$ & $10: 0$ & $10: 0$ & - & - & - \\
\hline V19-3 & $0: 10$ & $10: 0: 0: 0$ & $10: 0$ & $10: 0$ & $10: 0$ & $10: 0$ & - & - & - \\
\hline V23-1 & $0: 10$ & $10: 0: 0: 0$ & $9: 0$ & $\underline{6}: 4$ & $10: 0$ & $10: 0$ & + & - & + \\
\hline V24-1 & $0: 9$ & $9: 0: 0: 0$ & $0: 9$ & $9: 0$ & $0: 9$ & $9: 0$ & - & - & - \\
\hline V25-1 & $9: 0$ & 0:9:0:0 & $9: 0$ & $9: 0$ & $9: 0$ & $9: 0$ & - & - & - \\
\hline V29-1 & $\lfloor 0: 0$ & $0: 10: 0: 0$ & $10: 0$ & $\underline{5}: 5$ & $10: 0$ & $10: 0$ & + & - & + \\
\hline V30-1 & $0: 11$ & $6: \underline{5}: 0: 0$ & $\underline{7}: 4$ & $11: 0$ & $11: 0$ & $11: 0$ & + & + & + \\
\hline V33-1 & $0: 9$ & $0: 0: 9: 0$ & $0: 9$ & $\underline{3}: 6$ & $0: 9$ & $9: 0$ & + & - & + \\
\hline V33-2 & $0: 9$ & $0: 0: 9: 0$ & $0: 9$ & $9: 0$ & $0: 9$ & $9: 0$ & - & + & + \\
\hline V34-1 & $4: \underline{6}$ & $\underline{7}: 3: 0: 0$ & $10: 0$ & $10: 0$ & $10: 0$ & $10: 0$ & + & + & + \\
\hline V36-1 & $0: 10$ & $4: 6: 0: 0$ & $10: 0$ & $5: 5$ & $10: 0$ & $10: 0$ & + & + & + \\
\hline \multirow[t]{2}{*}{ V38-1 } & $0: 8$ & $8: 0: 0: 0$ & $8: 0$ & $8: 0$ & $8: 0$ & $8: 0$ & - & - & -1 \\
\hline & \multicolumn{6}{|c|}{ Total outcrosses discernible ${ }^{g}$} & 15 & 11 & 16 \\
\hline
\end{tabular}

${ }^{a}$ Discernible segregation $(+)$ for three types of genetic markers: RFLPs, vegetative compatibility $(\mathrm{VC})$ and DNA fingerprinting (fp).

${ }^{b}$ Numbers in the ratio are ordered from largest to smallest of the polymorphic restriction fragments or for the presence then absence of a fragment for $C B 15-3$.

${ }^{\mathrm{c}}$ Maternal genotype is underlined when segregation occurs.

${ }^{\mathrm{d}}$ Observed ratio is significantly different from $1: 1(P=0.035)$.

${ }^{\mathrm{e}}$ One of nine ascospore progeny was not compatible with the maternal strain. This ratio is significantly different from $1: 1$ $(P=0.020)$ and is interpreted as selfing with a mutation at one or more vic loci.

${ }^{\mathrm{f}}$ A novel restriction fragment was detected in 1 of 15 ascospore progeny and was considered to be a mutation.

g Total number of perithecia in which outcrossing is discernible for each type of genetic marker.

where $p_{\mathrm{ij}}$ is the frequency of the $i$ th allele at the $j$ th locus in the conidial pool, and $p_{\mathrm{m}, \mathrm{ij}}$ is the frequency of the $i$ th allele at the $j$ th locus in the maternal population. For estimates of $p_{\mathrm{i}}$ we used allele frequencies for the canker population $(N=39$, Table 1$)$; estimates of $p_{\mathrm{m}, \mathrm{i}}$ were determined for each locus from the maternal genotypes of the canker isolates for each perithecium $(N=22$, Table 4). The estimate for the probability of non-identification of an outcross event for this population was $\hat{\alpha}=0.082$. Using the equations in Shaw et al. (1981), we obtained the estimate $\hat{t}_{\mathrm{m}}=0.742$ with a S.E. of 0.108 . Thus, the multilocus estimate of $t$ is signifi- cantly less than 1.0, indicating a significant amount of self-fertilization occurring in this population.

The estimated value for $\hat{t}_{\mathrm{m}}$ is very close to the proportion of perithecia that were observed to be outcrossed using fingerprint data. There was segregation for at least one fingerprint fragment (Fig. 1) among progeny from 16 of the 22 perithecia; this proportion, 0.727 , is significantly less than $1.0(95$ per cent confidence interval $(0.54,0.91))$. Conversely, progeny for six perithecia ( 27.3 per cent) did not segregate for any fingerprint fragments. In one ascospore family, from perithecium V38-1, there was one progeny that had a 


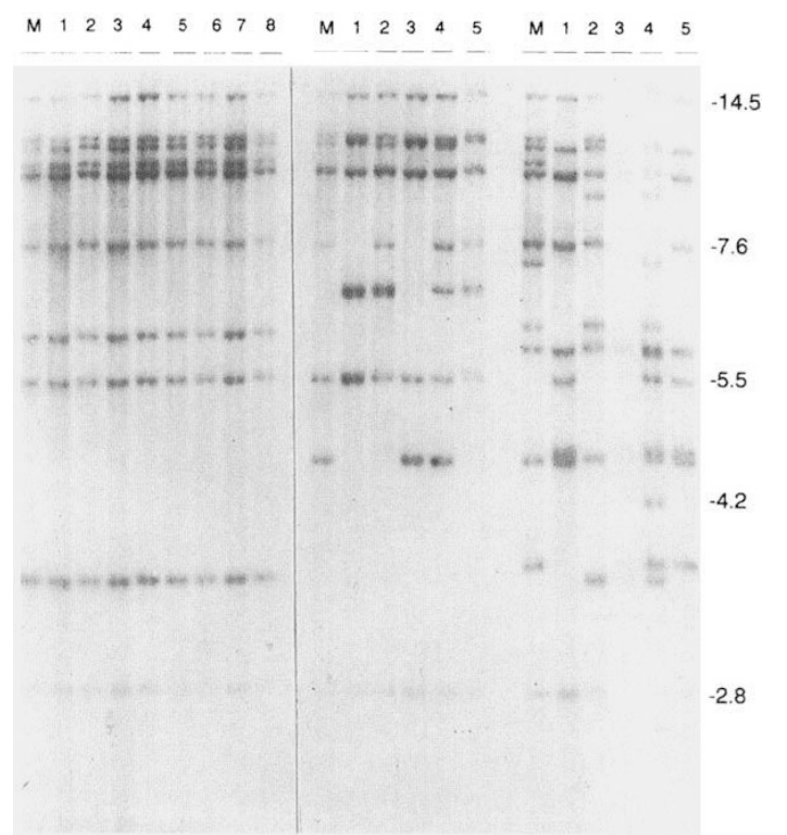

Fig. 1 DNA fingerprinting of three ascospore families with maternal canker isolates (lanes marked ' $M$ '). Segregation for several fragments was observed in the two families on the right, while no segregation was observed in the family on the left. See Milgroom et al. (1992a) for details on fingerprinting methods.

single novel fingerprint fragment We subsequently examined a total of 15 progeny from this family and found 14 lacking this fragment, concluding that mutation had occurred, not outcrossing. Using estimates of fingerprint fragment frequencies for the 39 canker isolates, $p_{\mathrm{i}}$ (conidial pool), and the subset of 22 maternal isolates, $p_{\mathrm{m}, \mathrm{i}}$ (Tables 3 and 4 in Milgroom et al., 1992a), we calculated the probability of failing to detect outcrosses as $\hat{\alpha}=0.000115$. Therefore, fingerprinting was a powerful marker for detecting all outcrosses with near certainty in this population.

In contrast to the fingerprint and RFLP data, vegetative compatibility segregated in only 11 of the 22 perithecia (Table 4). Progeny in 10 of the 11 perithecia that segregated for $\mathrm{VC}$ also segregated for fingerprinting and RFLPs; progeny from perithecium V33-2 segregated for $\mathrm{VC}$ and fingerprinting but not for RFLPs (Table 4). In perithecium V11-1 we found only one of nine progeny that was vegetatively incompatible with the maternal strain. This perithecium was scored as selfed as the probability of observing a single incompatible progeny when there is $1: 1$ segregation is 0.02 . We speculate that this incompatible progeny occurred by mutation and not by outcrossing. Regardless of how perithecium V11-1 is scored, VC data have limited value for estimation of outcrossing unless the frequency of each VC group is known and can be used to estimate the probability of non-identification of outcrosses.
The evidence that progeny from six perithecia are indeed the results of self-fertilization is strong as there is concordance between RFLP and fingerprint analyses for all but one perithecium (V33-2) (Table 4). This discrepancy is not disturbing because we expect approximately 8 per cent of the outcrosses to be nondiscernible with RFLP markers in this population (see above).

\section{Discussion}

Segregation data for RFLPs and DNA fingerprinting confirm that $C$. parasitica has a mixed mating system in a natural population. In this population, the outcrossing rate was estimated to be 0.74 by a multilocus estimation using RFLP data and 0.73 from the proportion of perithecia that are discernibly outcrossed as determined by fingerprint data. Another report of a quantitative estimate of the outcrossing rate for a fungus in a natural population was for Crumenulopsis sororia which exclusively outcrosses (Ennos \& Swales, 1987). We are not aware of any other reports in which fungal mating systems have been quantified in a natural population.

In contrast to $C$. sororia, we found no evidence that more than one conidial genotype was involved in fertilization to give rise to ascogenous hyphae within a single perithecium in $C$. parasitica. Laboratory studies with $C$. parasitica have shown that fertilization by more than one conidial nucleus is sometimes possible (Anagnostakis, 1982b). However, all but one of the perithecia had progeny that segregated in $1: 0,1: 1$ or $0: 1$ ratios for six independent loci, and there were no families with segregation of more than two alleles even when there were four alleles for locus MS26 present in this population. If multiple fertilization occurred in this population of $C$. parasitica, it was probably a rare event.

The fact that each perithecium appears to have been fertilized by a single conidial genotype led us to use perithecia as the units from which to estimate outcrossing rates. The probability of failing to detect outcrosses with fingerprints in this population was very small because there were 29 independent, polymorphic fingerprint loci in the maternal sample (Milgroom et al., 1992a). Therefore, fingerprinting detected virtually all the outcrosses and the proportion of outcrossed perithecia is equivalent to the outcross rate for this population. The fact that the multilocus estimate of outcrossing rate, $\hat{t}_{\mathrm{m}}$, based on six RFLP loci was almost identical to the proportion of outcrossed perithecia determined by fingerprinting, is partly because the six RFLP loci were diverse enough in this population that we would expect to detect approximately 92 per cent 
of the outcrosses; 15 of the 16 outcrosses detected by fingerprinting ( 94 per cent) were in fact detected by RFLPs.

One assumption in the multilocus estimate of outcrossing is that mating occurs randomly (Shaw et al., 1981). That is, the allele frequencies in the conidial pool (male parents) are identical for all maternal isolates. This assumption may not be appropriate because conidia of $C$. parasitica are dispersed by rain and animals (see Anagnostakis, 1987) which, unlike wind dispersal, may result in relatively short-distance and non-random dispersal patterns. A consequence of short-distance dispersal of conidia is that relatively few genotypes can serve as male parents for each perithecium. Therefore, there is some potential for violation of the random mating assumption if there is spatial genetic structure in the population. Ennos \& Swales (1987) also suggested short-distance dispersal of male gametes in Crumenulopsis sororia as a possible cause of non-random mating. In addition, they pointed out that with mating incompatibility systems, such as those found in C. parasitica (Anagnostakis, 1988), the potential number of males for each fruiting body is reduced even further. This problem of limited dispersal of male gametes has been recognized with plant mating systems where population subdivision causes estimates of $t$ to be biased downward simply because of differences in the effective pollen pool for each individual in a population (Brown et al., 1975). In the present study, however, we do not have sufficient data to test for population subdivision. However, the hypothesis of random mating was not rejected in this population when isolates with identical fingerprint genotypes were eliminated from the analysis (Milgroom et al., 1992a). The fact that $\hat{t}_{\mathrm{m}}$ was almost identical to the proportion of perithecia with segregation for fingerprints, may be another indication that violations of the random mating assumption are not severe in this population.

The proportion of outcrossed perithecia discernible with vegetative compatibility was much less than the proportion discernible with fingerprinting or RFLPs. One possible explanation for this lower proportion is that the diversity of VC groups, unlike RFLPs and fingerprints, is not great enough in this population to detect a large fraction of the outcrosses. Although VC diversity may be high in some $C$. parasitica populations (MacDonald \& Double, 1978; Kuhlman \& Bhattacharyya, 1984; Anagnostakis \& Kranz, 1987; Milgroom et al., 1991), some VC groups may be relatively common. For example, more than a third of all isolates from cankers sampled over 3 years in a population in Connecticut were in a single VC group (Anagnostakis \& Kranz, 1987). Furthermore, it is also known that there can be considerable fingerprint diversity within
VC groups (Liu \& Milgroom, 1992; Y.-C. Liu \& M. G. Milgroom, unpublished data) so that crosses between members of the same VC group can show segregation for fingerprints but not for VC. In the absence of VC group frequency data from this population, however, this explanation is only speculative.

There are a number of further questions that should be investigated about the mating system of $C$. parasitica, e.g. the ecological factors affecting outcrossing rates, such as environmental conditions or population density, or whether outcrossing rates vary among different genotypes. This study was conducted on only a single sample. There is some indication that outcrossing rates may vary in different populations (M. G. Milgroom \& S. E. Lipari, unpublished data) or in different years and ages of cankers (S. L. Anagnostakis, personal communication). Better knowledge of the mating system of $C$. parasitica is important for understanding the population structure and evolution of this pathogen and may eventually provide some insights for implementing biological control (MacDonald \& Fulbright, 1991) of this fungal pathogen.

\section{Acknowledgements}

We thank R. Vilgalys for assistance in collecting at Mt. Lake, and S. L. Anagnostakis for sharing unpublished data. This research was supported in part by the United States Department of Agriculture competitive grant 91-37303-5939.

\section{References}

ADAMS, G., HAMMER, S. AND PROFFER, T. 1990. Vegetative compatibility in Leucostoma persoonii. Phytopathology, 80, 287-291.

AINSWORTH, A. M. 1987. Occurrence and interactions of outcrossing and non-outcrossing populations in Stereum, Phanerochaete and Coniophora. In: Rayner, A. D. M., Brasier, C. M. and Moore, D. (eds) Evolutionary Biology of the Fungi, Cambridge University Press, Cambridge.

ALLARD, R. w. 1975. The mating system and microevolution. Genetics, 79, 115-126.

ANAGNOSTAKIS, S. L. 1982a. Genetic analyses of Endothia parasitica, linkage data for four single genes and three vegetative compatibility types. Genetics, 102, 25-28.

ANAGNOSTAKIS, S. L. 1982 b. The origin of ascogenous nuclei in Endothia parasitica. Genetics, 100, 413-416.

ANAGNOSTAKIS, S. L. 1987. Chestnut blight, the classical problem of an introduced pathogen. Mycologia, 79, 23-37.

ANAGNOSTAKIS, s. L. 1988. Cryphonectria parasitica cause of chestnut blight. Adv. Plant Path., 6, 123-136.

ANAGNOSTAKIS, S. L. AND KRANZ, J. 1987. Population dynamics of Cryphonectria parasitica in a mixed-hardwood forest in Connecticut. Phytopathology, 77, 751-754. 
BERAHA, L. AND GARBER, E. D. 1985. Relative homothallism and production of hybrid perithecia by auxotrophic mutants of Glomerella cingulata from apple. Phytopath. Z., 112, 32-39.

BRASIER, C. M. AND GIBBS, J. N. 1975. Highly fertile form of the aggressive strain of Ceratocystis ulmi. Nature, 257, 128-131.

BROWN, A. H. D., MATHESON, A. C. AND ELDRIDGE, K. G. 1975. Estimation of the mating system of Eucalyptus obliqua L'Herit by using allozyme polymorphisms. Aust. J. Bot., 23, 931-949.

CLEGG, M. T. 1980. Measuring plant mating systems. BioScience, 30, 814-818.

ENNOS, R. A. AND SWALES, K. w. 1987. Estimation of the mating system in a fungal pathogen Crumenulopsis sororia (Karst.) Groves using isozyme markers. Heredity, 59, 423-430.

FINCHAM, J. R. S., DAY, P. R. AND RADFORd, A. 1979. Fungal Genetics, 4th edn. University of California Press, Berkeley. HEDRICK, P. W. 1990. The mating system and evolutionary genetics. In: Wohrmann, K. and Jain, S. (eds) Population Biology, Ecological and Evolutionary Viewpoints, Springer, Berlin.

HEMMONS, L. M., PONTECORVo, G. AND BuFTON, A. W. 1958. Perithecium analysis and relative homothallism. $A d v$. Genet., 5, 194-201.

KOHLI, Y. AND KOHN, L. M. 1992. In vitro and in vivo outbreeding studies on Sclerotinia sclerotiorum. Newsl. Mycol. Soc. Am., 43, 39 (abstract).

KOHN, L. M., STASOVSKI, E., CARBONE, I., ROYER, J. AND ANDERSON, J. B. 1991. Mycelial incompatibility and molecular markers identify genetic variability in field populations of Sclerotinia sclerotiorum. Phytopathology, 81, 480-485.

KUHLMAN, E. G. AND BHATTACHARYYA, H. 1984. Vegetative compatibility and hypovirulence conversion among naturally occurring isolates of Cryphonectria parasitica. Phytopathology, 74, 659-664.

LIU, Y.-C. AND MILGROOM, M. G. 1992. Analysis of genetic diversity within vegetative compatibility groups of Cryphonectria parasitica using DNA fingerprinting. Phytopathology, $\mathbf{8 2}, 245$ (abstract).

MACDONALD, W. L. AND DOUBLE, M. L. 1978. Frequency of vegetative compatibility types of Endothia parasitica in two areas of West Virginia. In: MacDonald, W. L., Cech,
F. C., Luchok, J. and Smith, C. (eds) Proceedings of American Chestnut Symposium, West Virginia University Books, Morgantown, WV.

MACDONALD, W. L. AND FULBRIGHT, D. W. 1991. Biological control of chestnut blight: use and limitations of transmissible hypovirulence. Plant Dis., 75, 656-661.

MILGROOM, M. G., LIPARI, S. E. AND POWELl, w. A. 1992a. DNA fingerprinting and analysis of population structure in the chestnut blight fungus, Cryphonectria parasitica. Genetics, 131, 297-306.

MILGROOM, M. G., LIPARI, S. E. AND WANG, K. 1992b. Comparison of genetic diversity in the chestnut blight fungus, Cryphonectria (Endothia) parasitica, from China and the US. Mycol. Res., 96, 1114-1120.

MILGROOM, M. G., MACDONALD, W. L. AND DOUBLE, M. L. 1991. Spatial pattern analysis of vegetative compatibility groups in the chestnut blight fungus, Cryphonectria parasitica. Can. J. Bot., 69, 1407-1413.

POWELL, W. A. AND VAN ALFEN, N. K. 1987. Differential accumulation of $\operatorname{poly}(\mathrm{A})+\mathrm{RNA}$ between virulent and doublestranded RNA-induced hypovirulent strains of Cryphonectria (Endothia) parasitica. Mol. Cell. Biol., 7, 3688-3693.

PUHAlla, J. E. AND ANAgnostakis, s. L. 1971. Genetics and nutritional requirements of Endothia parasitica. Phytopathology, 61, 169-173.

RAYNER, A. D. M. AND TURTON, M. N. 1982. Mycelial interactions and population structure in the genus Stereum, $S$. rugosum, S. sanguinolentum and S. rameale. Trans. Br. Mycol. Soc., 78, 483-493.

SHARLAND, P. R. AND RAYNER, A. D. M. 1989. Mycelial ontogeny and interactions in non-outcrossing populations of Hypoxylon. Mycol. Res., 93, 273-281.

SHARLAND, P. R. AND RAYNER, A. D. M. 1989. Mycelial interactions in outcrossing populations of Hypoxylon. Mycol. Res., 93, 187-198.

SHARLAND, P. R., RAYNER, A. D. M., OFONG, A. U. AND BARRETT, D. K. 1988. Population structure of Rosellina desmatzieresii causing ring-dying in Salix replus. Trans. Br. Mycol. Soc., 90, 654-656.

SHAW, D. V., KAHLER, A. L. AND ALLARD, R. W. 1981. A multilocus estimator of mating system parameters in plant populations. Proc. Natl. Acad. Sci. U.S.A., 78, 1298-1302. 